\title{
Recurrent pathological fractures in osteitis fibrosa cystica: a case of undetected primary hyperparathyroidism due to parathyroid adenoma
}

\author{
W.Y.M. Abeysekera, MBBS ${ }^{1}$, P.M.C Udaya Kumara, MBBS MD², A.A.W.K. Amarasinghe, MBBS \\ $\mathrm{MD}^{2}$, D.M.H.Fernando, MBBS ${ }^{1}$, A.B.S. Ananda Perera, MS FRCS 3 \\ 1 Registrar in Orthopaedics, Sri Jayawardenepura General Hospital, Kotte, Sri Lanka. \\ 2 Senior Registrar in Orthopaedics, Sri Jayawardenepura General Hospital, Kotte, Sri Lanka. \\ 3 Consultant Orthopaedic Surgeon, Sri Jayawardenepura General Hospital, Kotte, Sri Lanka.
}

Key words: Osteitis fibrosa cystica; Primary hyperparathyroidism; Parathyroid adenoma; Brown tumour.

\section{Introduction}

Osteitis fibrosa cystica (OFC) is a skeletal disorder which results in exposure to high levels of parathormone (PTH), mainly due to untreated primary hyper-parathyroidism (PHPT). This is a case of primary hyperparathyroidism detected in a patient investigated for pathological fractures. Since this is a treatable condition early detection and treatment by routine assessment of serum calcium and phosphate levels in the aged population must be emphasised especially in developing countries.

\section{Key Learning Points}

Early detection and treatment in Primary Hyper-parathyroidism will prevent the disease progression into Osteitis Fibrosa Cystica

Serum calcium and phosphate levels should be done as routine investigations in elderly patients presenting with fractures

\section{Case}

A 58 year old female presented with a left supracondylar fracture of the humerus (Figure 1) following an accidental fall on her outstretched hand. She had had a subtrochanteric fracture of the right femur five years back, which had been fixed with a K nail. (Figure 2). She had a goiter which was only visible on neck extension.

$\mathrm{X}$ rays of the left arm and the right lower limb revealed large osteolytic bony lesions in the left distal humerus (Figure 1) and the left middle third of the tibia (Figure 3). In the hand X-ray, subperiosteal resorption of phalangeal

\footnotetext{
Correspondence: Dr. W.Y.M Abeysekera

No. 10A, Mission lane,

Pita-Kotte, Kotte,

Colombo, Srilanka

E-mail: yohan.abeysekera@yahoo.com
}

bones was evident (Figure 4).

Biochemical investigations revealed elevated levels of alakaline phosphatase (ALP), high serum total and ionised calcium levels, increased parathyroid hormone (PTH) levels and low phosphate levels were noted. Her thyroid functions were normal. Ultrasound scan of the neck revealed a multi-nodular goitre with a hyperechoic nodule in the infero-posterior aspect of the left lobe of thyroid raising the possibility of a parathyroid adenoma and contrast CT scan of the neck confirmed the left parathyroid adenoma and the multiple heterogenous enhancing thyroid nodules. Osteitis fibrosa cystica due to primary hyperparathyroidism was diagnosed.

She underwent a left hemithyroidectomy with left parathyroidectomy. Histology revealed a parathyroid adenoma. Following surgery the serum calcium level demonstrated a rapid drop and calcitriol with calcium lactate supplements were needed to maintain normal serum calcium levels.

\section{Discussion}

PHPT is a condition with a female preponderance and the peak incidence will be at age 30-50 years. One third of PHPT patients are asymptomatic. The annual incidence is $2-10 / 10000$ and nearly $60 \%$ of them will be identified during routine biochemical investigations [1].

Majority of the PHPT cases are due to solitary or multiple parathyroid adenomas. Only 20\% would be due to parathyroid carcinoma, hereditary causes like MEN type I/IIa/IIb or renal osteodystrophy [2].

Elevated PTH levels will stimulate bone resorption via high osteoclastic activity, mobilising large amounts of calcium in to the blood stream. Thus, the altered calcium metabolism will lead to renal calculi, peptic ulcers, loss of appetite, loss of weight and weak bones which is referred to as 'bones, stones, abdominal groans and psychic moans' [3].

Elevated serum levels of calcium, PTH and ALP, low phosphate levels with radiological evidence of bone 
resorption are characteristic findings in PHPT. The first bones to show subperiosteal erosions are the bones of the hand. Characteristic X-ray changes in the skull are described as ground glass or salt and pepper appearance [4].

Osteitis fibrosa cystica also known as osteitis fibrosa, osteodystrophia fibrosa, Von Recklinghausen's disease of bone, is a skeletal disorder caused by prolonged exposure of bone to elevated PTH levels in PHPT. Gerhard Engel in 1864 and Friedrich Daniel von Recklinghausen in 1890 described this condition for the first time [5].

The progression of PHPT to OFC is relatively uncommon in developed countries during the past few decades due to the improved techniques in early detection and treatment of the condition [3].

But still it is not uncommon in developing countries as a result of failure in early diagnosis and treatment or poor patient compliance.

OFC is evident in X-ray films as multiple cystic lesions in bones. These are known as 'brown tumours'. Brown tumours are benign hypervascular focal lesions in bones caused by increased osteoclastic activity and fibroblastic proliferation [6]. They are seen commonly in facial bones, mandible, sternum, pelvis, ribs, femurand rarely in vertebrae [6]. Even though pathological fractures in PHPT are relatively uncommon [7], areas of bone with brown tumours are vulnerable to fractures with trivial trauma.

The treatment of choice would be total para-thyroidectomy. It has been shown to result in the reversal of bone resorption and the complete regression of brown tumours [8]. According to some reports, total para-thyroidectomy, even in parathyroid carcinoma will be the remedy to control the symptoms of hyperparathyroidism and OFC [9]. Bone transplants can also be used to fill the lesions (cavities) caused by OFC [10].

\section{References}

1. Morgan G, GanapathiM, Afzal S, Grant AJ. Pathological fractures in primary hyperparathyroidism: a case report highlighting diagnostic difficulties. Injury 2002; 33(3): 288-91.

Doi: $\quad$ http://dx.doi.org/10.1016/S0020-

1383(01)00134-6

PMID: 12084655
2. Maina AM, Kraus H. Successful Treatment of Osteitis Fibrosa Cystica from Primary Hyperparathyroidism. Case Reports in Orthopedics. 2012; 2012. Doi:10.1155/2012/145760

3. Favus MJ, Primer on the Metabolic Bone Diseases and Disorders of Mineral Metabolism, 1993; 2. PMCid:288005

4. Rubin MR, Livolsi VA, Bandeira F, Caldas G, Bilezikian JP. Clinical case seminar: Tc99m-sestamibi uptake in osteitis fibrosa cystica simulating metastatic bone disease, Journal of Clinical Endocrinology and Metabolism, 2001; 86(11): 5138-41.

Doi: http://dx.doi.org/10.1210/jc.86.11.5138 PMid:11701666

5. Buchanan WW, Kraag GR, Palmer DG, Cockshott WP. Canadian Medical Association journal 1981; 124(6): 812-5. PMid:7471024 PMCid:1705307

6. Khaoula BA, Kaouther BA, Ines C, Sami T, Zakraoui $\mathrm{L}$, et al. An Unusual Presentation of Primary Hyperparathyroidism: Pathological Fracture. Case Reports in Orthopedics 2011; 2011: 521-78.

Doi: 10.1155/2011/521578 PMCID: PMC3505895

7. Chalmers J, Irvine GB. Fractures of the femoral neck in elderly patients with hyperparathyroidism.Clinical Orthopaedic Related Research.1988; 229: 125-30. PMID: 3349668

8. Arabi A, Khoury N, Zahed L, Birbari A, El-Hajj Fuleihan G. Rgression of Skeletal Manifestations of Hyperparathyroidism with Oral Vitamin D. Journal of Clinical Endocrinology \& Metabolism. 2006; 91(7): 2480-3.

Doi: http://dx.doi.org/10.1210/jc.2005-2518 PMid:16608887

9. Ellis HA, Floyd M, Herbert FK. Recurrent hyperparathyroidism due to parathyroid carcinoma. Journal of Clinical Pathology 1971; 24(7): 596-604.

Doi: http://dx.doi.org/10.1136/jcp.24.7.596 PMid:4107487 PMCid:477103

10. Brand, Richard A. Clinical Orthopaedics and Related Research. 2008; 466(1): 22-36. 


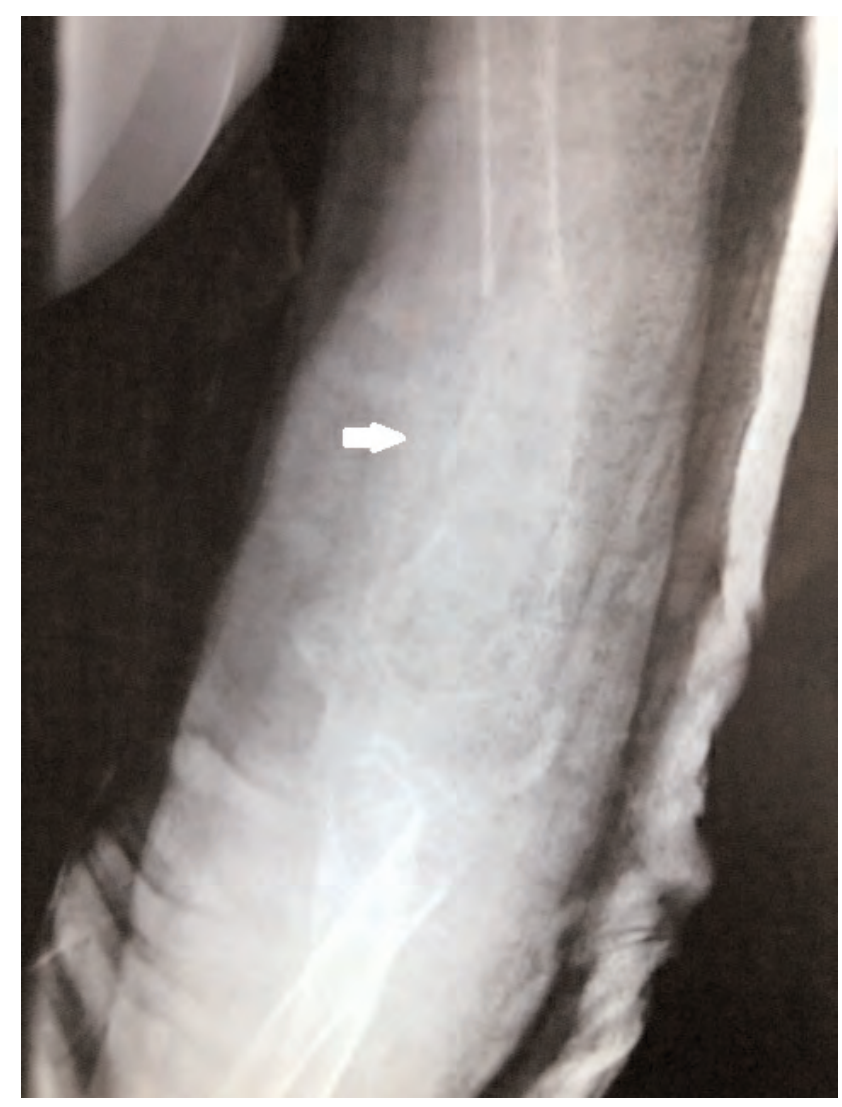

Figure 1. White arrow indicates a left supracondylar fracture of the humerus at the site of a large cystic lesion of the bone (brown tumour), managed with manipulation under anesthesia and plaster cast.

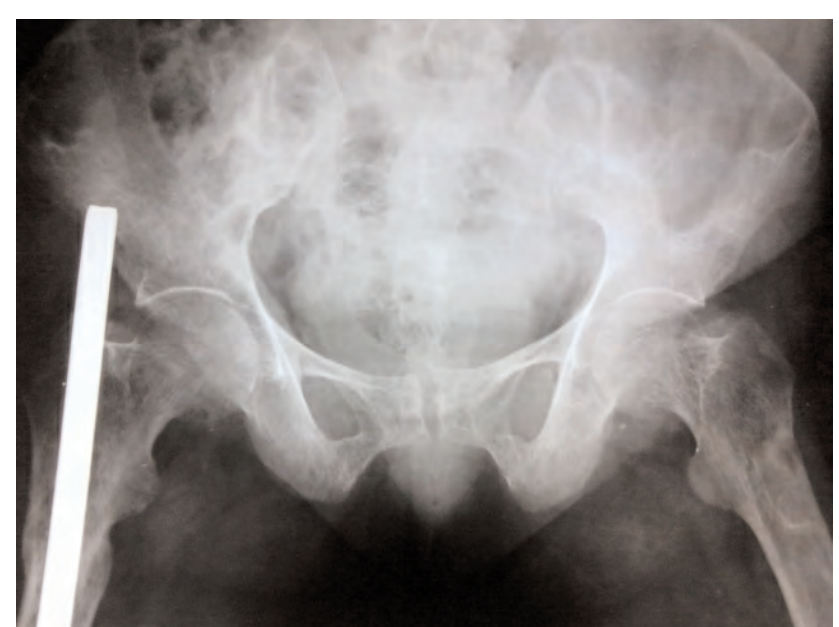

Figure 2. Right femur sub-trochanteric fracture fixed with a K-Nail 


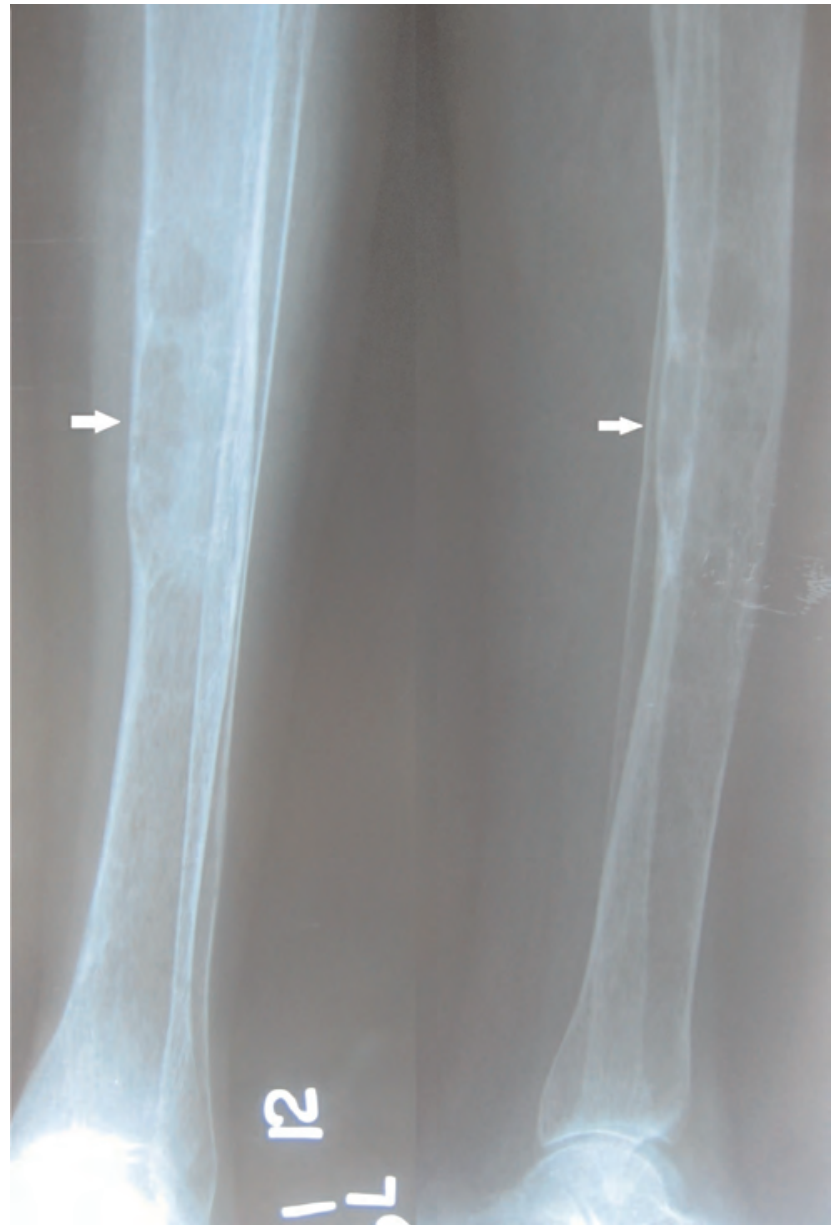

Figure 3. White arrows indicate large osteolytic bony lesion in the left middle third of the tibia (brown tumour) in AP and lateral X-ray views of the left leg.

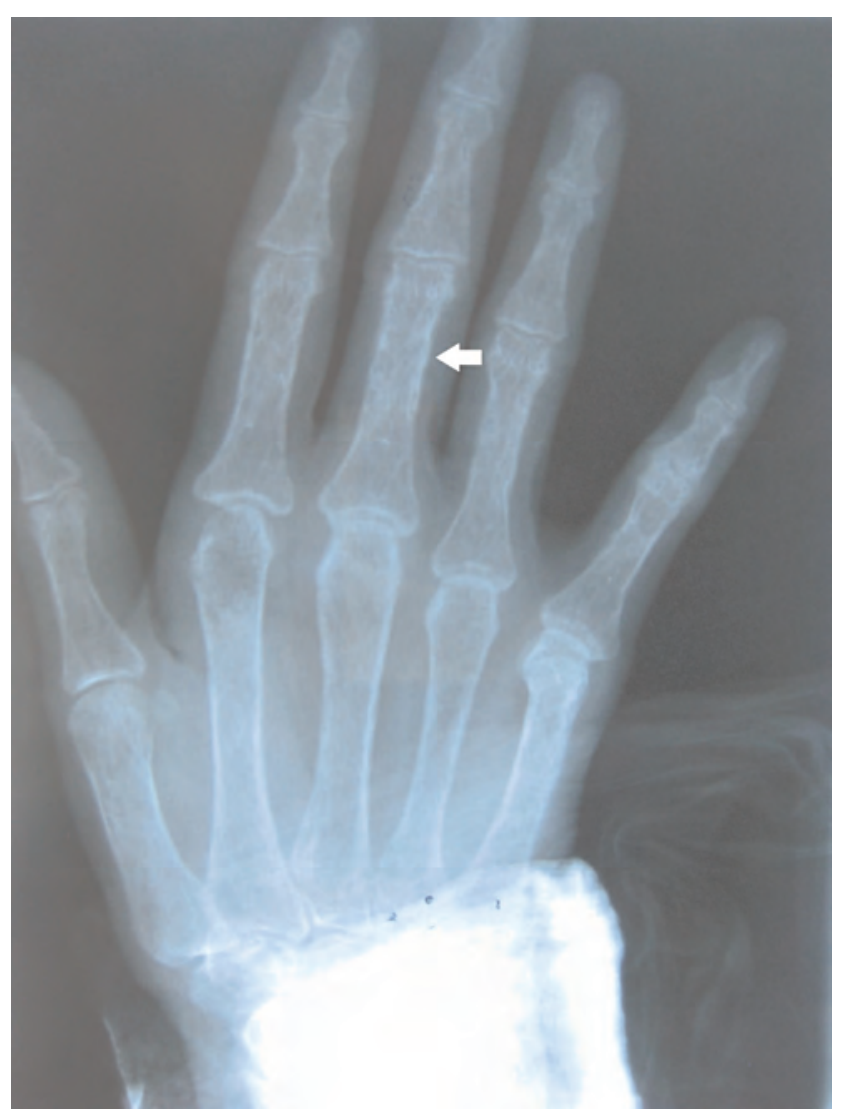

Figure 4. White arrow indicates subperiosteal resorption of phalangeal bones. 